\title{
Immunogenicity of a Combined DTPa-HB Vaccine Co-Administered with Haemophilus influenzae Type B Conjugate Vaccine (PRP-T) for Primary and Booster Vaccinations
}

Humberto Bracco Neto, Anete Colucci, Rosana F. Puccini and Calil K. Farhat
Department of Pediatrics - Federal University of São Paulo UNIFESP; São Paulo, SP, Brazil

\begin{abstract}
Objective: To evaluate the immunogenicity of a combined DTPa-HB vaccine co-administered with Haemophilus influenzae type b conjugate vaccine (PRP-T) in Brazilian infants. Material and Methods: A prospective and open clinical study, in which 110 infants were immunized with a threedose primary vaccination regime at two, four and six months of age and with a single booster vaccination. Blood samples were drawn immediately before the first dose, one month after the third dose, at the time of the booster dose and one month after the booster to assess seropositivity and antibody geometric mean titers (GMTs) of antibodies for diphtheria, tetanus, hepatitis B, Haemophilus influenzae type $b$ and for the three pertussis antigens: Pertussis Toxin (PT), Filamentous Hemagglutinin (FHA) and Pertactin (PRN). Results: Among the original 110 infants, 93 completed the study. Seropositivity was $100 \%$ for all seven involved antibodies, after the primary vaccination course. At the time of the booster dose, all antibodies (except diphtheria $33.7 \%$ and anti-PT $59 \%$ ) were seropositive for more than $94 \%$ of subjects. After the booster, seropositivity increased to $\mathbf{1 0 0} \%$ for all antibodies. The GMT of these antibodies followed a similar pattern, with a strong increase after the primary course, followed by a second increase after the booster dose. At this time, GMT was2- to 7-fold higher than after the primary course, for all vaccine components. Conclusions: Concomitant administration of DTPa-HB and Hib vaccines elicited strong seroprotection for all the antigenic components. No interference with antibody response was evident. The vaccines provided high immunogenicity, following both the primary vaccinations and the booster dose.

Key Words: Vaccines/immunology; vaccines, combined, diphtheria-tetanus-acellular pertussis vaccines, hepatitis B vaccines, Haemophilus vaccines.
\end{abstract}

The development of immunology and molecular biology has, over recent years, promoted impressive growth in the number of vaccines available [1]. Current practice in pediatric vaccination is to stimulate the utilization of vaccine combinations and concomitant administration of vaccines, with the aims of diminishing discomfort among vaccination patients and facilitating adherence to the immunization scheme [2,3].

Received on 15 April 2005; revised 7 August 2005.

Address for correspondence: Dr. Humberto Bracco Neto. Rua Correa de Lemos 780 apto 183 B, Zip code: 04140-000 Phone: (011) 5072-4287. Fax (011) 5055-1891 E-Mail humberto@imunoclin.com.br.

The Brazilian Journal of Infectious Diseases 2005;9(5):363-373 (C) 2005 by The Brazilian Journal of Infectious Diseases and Contexto Publishing. All rights reserved.
Inclusion of the vaccine against hepatitis B as a component of a combined vaccine has the convenience of improving adherence and consequently the vaccination coverage. This is considered crucial for reducing the dissemination of hepatitis $B$ and the associated morbidity and mortality [3,4]. The Pertussis vaccine combined with Diphtheria and Tetanus toxoids, the most widely disseminated and successful combined vaccine, has served as the basis for most vaccine combinations and presents the acellular pertussis component as an alternative to traditional vaccines that use whole cells, with similar efficacy and lower reactogenicity [5]. Finally, combined or concomitant administration of conjugated vaccine against Haemophilus influenzae type b with other vaccines 
has taken on fundamental importance, as demonstrated by the significant reduction in the incidence of the disease seen in countries that have incorporated it into their vaccination calendars [6,7].

Despite all the advantages of combined vaccines, the physical, chemical or biological properties of each vaccine component may interfere with the other components. This may affect the stability, efficacy or safety of the vaccine combination [3,8]. Moreover, different populations may present varying responses with regard to immunogenicity $[9,10]$. Thus, it is important to determine whether or not the combined or concomitant administration of several vaccines diminishes the specific response for each vaccine antigen, and to evaluate the resultant effect of including one or more vaccine components in combination with a vaccine that previously has been proven efficacious in the immunogenicity of its components.

We examined the immunogenicity of a diphtheriatetanus-acellular pertussis vaccine (DTPa), combined with the vaccine against hepatitis B (HB), and of a vaccine against Haemophilus influenzae type b (PRPT), administered simultaneously to Brazilian children.

\section{Material and Methods}

This study was preceded by approval from the National Commission for Research Ethics (Comissão Nacional de Ética em Pesquisa - CONEP) and from the Research Ethics Committees of Unifesp/Hospital São Paulo and the Health Department of the municipality of Embu, state of São Paulo. It was developed at the Healthcare Unit of Jardim Santo Eduardo, in the municipality of Embu, which is one of the units in which the activities of the pediatrics team of the Teaching-Attendance Integration Program of the Federal University of São Paulo (Unifesp) are concentrated. Written informed consent was obtained from the parent or guardian of each subject prior to entry into the study.

This was a prospective, open-label, single group clinical study of the immunogenicity of a combined diphtheria, tetanus, acellular pertussis and recombinant
Hepatitis B vaccine co-administered with Haemophilus influenzae type b (Hib) vaccine as a primary vaccination course in 110 healthy infants aged 2, 4 and 6 months, followed by a booster administered between 15 and 18 months of age. To investigate the immunogenicity of the vaccines, blood samples were drawn immediately before the first dose, one month after the third dose, at the time of the booster dose and one month after the booster (Figure 1).

Inclusion criteria were infants between 6 and 10 weeks of age at the time of the first vaccination, with written informed consent obtained from the parents or guardians of the subject. Exclusion criteria were history of allergic disease likely to be stimulated by the vaccination, history of progressive neurological disease, immunosuppressive therapy, any suspected or confirmed immunosuppressive or immunodeficient condition, immunoglobulin therapy or administration of any blood product since birth or during the study period. The vaccines used in this study were developed and manufactured by SmithKline Beecham Biologicals (Rixensart, Belgium). Acombined diphtheria, tetanus, acellular pertussis, and hepatitis B vaccine (DTPa$\mathrm{HBV}$ ) was supplied in a monodose vial. One dose (0.5 $\mathrm{mL}$ ) of the combined DTPa-HBV contained: $25 \mu \mathrm{g}$ pertussis toxin (PT), $25 \mu \mathrm{g}$ filamentous haemagglutinin (FHA), $8 \mu$ g pertactin (PRN), at least $30 \mathrm{IU}$ (international units) Diphtheria toxoid, at least $40 \mathrm{IU}$ Tetanus toxoid, $10 \mu$ g recombinant Hepatitis B surface antigen (HbsAg), $0.7 \mathrm{mg}$ aluminum (as a salt) and 2.5 mg phenoxyethanol. The Haemophilus influenzae type b conjugate vaccine (Hib) was presented as a freeze-dried pellet in a monodose vial to be reconstituted before use with a sterile saline diluent (9 $\mathrm{mg} \mathrm{NaCl} / \mathrm{mL})$, and one dose $(5 \mathrm{~mL})$ contained $10 \mu \mathrm{g}$ Polyribosyl-Ribitol-Phosphate, between 20 and $40 \mu \mathrm{g}$ Tetanus toxoid and $12.6 \mathrm{mg}$ lactose. The vaccines were administered as separate, concomitant, deep intramuscular injections in opposite limbs into the anterolateral part of the thigh.

Blood samples were centrifuged and separated. All serum samples were stored at $-20^{\circ} \mathrm{C}$ and shipped for testing at SmithKline Beecham Biologicals laboratory in Rixensart, Belgium. Antibodies against each vaccine 
antigen were determined by serological assays, calibrated with international standards. Antibodies against diphtheria, tetanus, pertussis components (PT, FHA, PRN) and Hib (PRP) were determined by enzyme-linked immunosorbent assay (ELISA). Antidiphtheria and anti-tetanus titers were expressed in IU/ $\mathrm{mL}$ and calibrated against the WHO reference serum [11]. It is generally accepted for both antibodies that titers greater than $0.01 \mathrm{IU} / \mathrm{mL}$, measured by in vivo neutralization tests, are protective. Agood correlation exists between neutralization tests and the ELISAs, but this correlation may be reduced at titers lower than 0.1 $\mathrm{IU} / \mathrm{mL}$ [12]. A titer of $0.1 \mathrm{IU} / \mathrm{mL}$ was therefore conservatively used as the minimum titer indicative of protection. Antibodies against PT, FHA and PRN antigens were calibrated with the Food and Drug Administration(FDA) reference serum, considered an international standard [13], and the cut-off for all three pertussis antibodies was set at 5 enzyme-linked immunosorbent assay units (EL.U)/mL. The anti-PRP antibody concentration of $0.15 \mu \mathrm{g} / \mathrm{mL}$ was set as the cut-off. A serum concentration greater than $1 \mu \mathrm{g} / \mathrm{mL}$ was also considered predictive of long-term protection against Hib invasive disease. Antibodies against Hepatitis B surface antigen were measured in milliInternational Units (mIU/mL) by radioimmunoassay (AUSAB, Abott Laboratories), titered against a WHO reference standard. The minimum antibody titer necessary for protection against hepatitis B was considered to be $10 \mathrm{mIU} / \mathrm{mL}$, which is generally accepted to be protective [14].

Seropositivity rate was defined as the proportion of subjects with antibody titers greater than or equal to the laboratory-defined assay cut-off value. The GMT, with a 95\% confidence interval (CI), was calculated by taking the anti-log of the mean of the log-titer transformations. Antibody titers below the cut-off level were given an arbitrary value of half the cut-off value for the purpose of GMT calculation. The data were transcribed onto electronic media and processed on an IBM-compatible microcomputer. The database management and statistical analyses were performing using Statistical Analysis Systems (SAS) 6.12 and StatXact-3, both on Windows NT 4.0.

\section{Results}

Out of the 110 children who began the follow-up, 93 concluded the study 17 months later, thus representing a loss of $15.5 \%$. No child had any serious adverse reaction, and all such occurrences have been described in a specific study [15].

The seropositivity rates were determined for the diphtheria and tetanus antigens at the four serum collection times (Figure 2). For the diphtheria component, more than $60 \%$ of the children already presented antibody concentrations of more than $0.1 \mathrm{IU} / \mathrm{mL}$ before starting the vaccination. One month after concluding the basic scheme, all of the children had reached concentrations greater than the cut-off value. Immediately before the booster dose, the seropositivity rate was found to have fallen considerably, down to around 30\%. There was a return to $100 \%$ seropositivity among the children after the booster dose. The seropositivity rate against the tetanus antigen before starting the vaccination was also close to $60 \%$. After the basic scheme, $100 \%$ of the children reached protective levels. At the time of the booster dose, this rate had fallen slightly, but it returned to $100 \%$ after the booster.

The seropositivity rate was determined for the antigens of the pertussis component (Figure 3). Before starting the vaccination, the filamentous hemagglutinin antigen(FHA) gave the highest seropositivity, with more than $90 \%$ of the children already seropositive at that time. After the primary immunization, the three antigens (pertussis toxin, FHA and pertactin) attained a seropositivity rate of $100 \%$. Before the booster dose, the seropositivity for the pertussis toxin (PT) had fallen to a rate of less than $60 \%$. The other two antigens presented rates of close to $100 \%$ at this time. After the booster dose, the seropositivity rate again reached $100 \%$ for the three antigens.

The seropositivity rate for the Hepatitis B surface antigen (HbsAg) was determined (Figure 4). Before starting the vaccination, less than $2 \%$ of the children were seropositive. After the basic scheme, $100 \%$ of the children attained protective levels. This percentage had fallen slightly by the time of the booster dose, and it returned to $100 \%$ after this dose. 
Figure 1. Schematic representation of the study schedule.

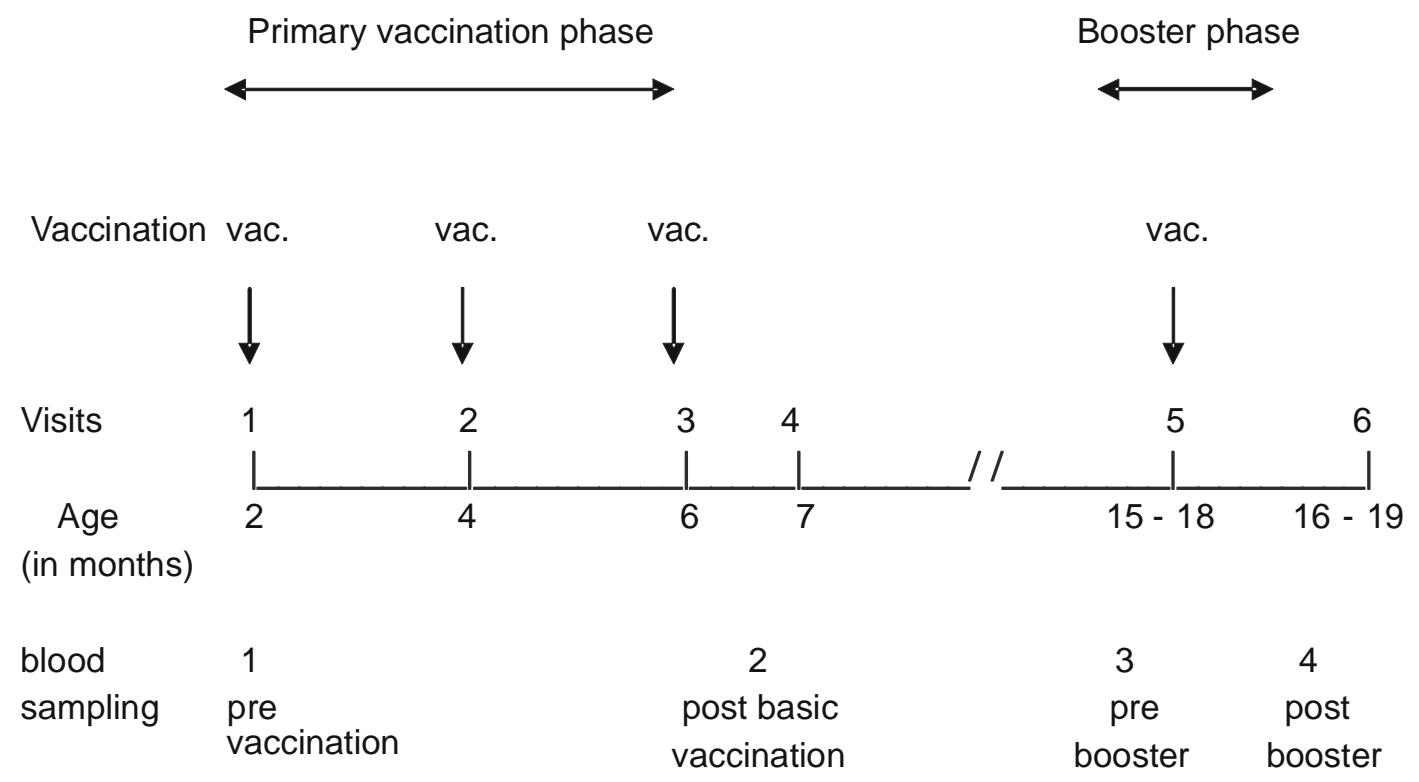

Figure 2. Seropositivity rate for diphtheric and tetanic antigens, according to the blood sampling time point.

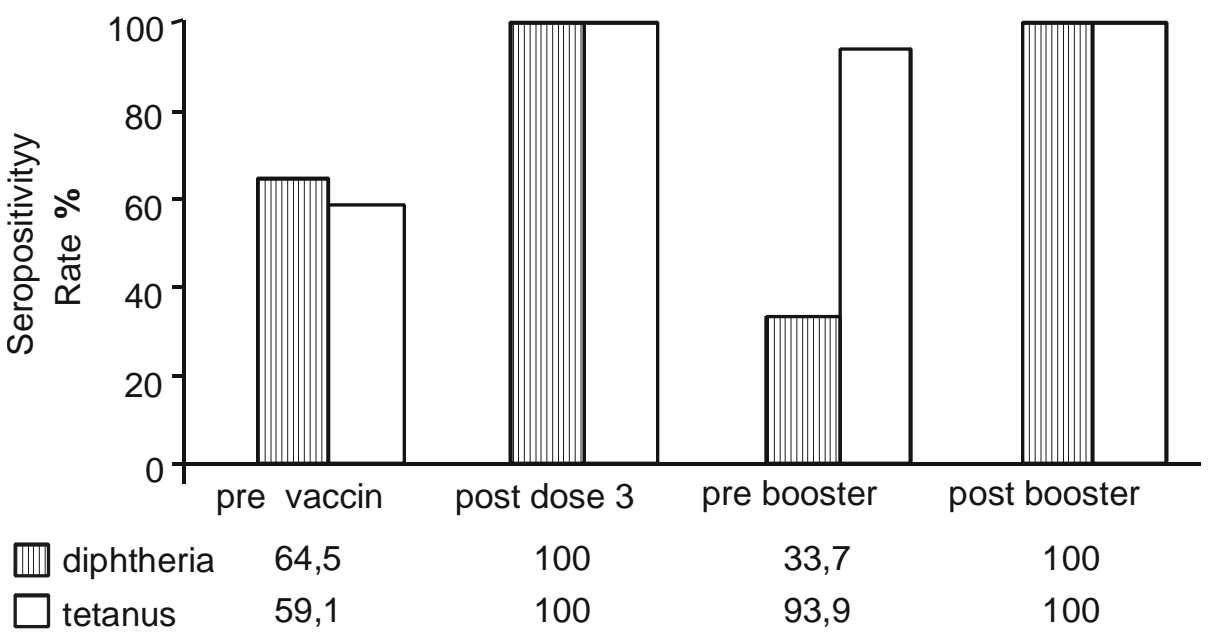


Figure 3. Seropositivity rate for the three Pertussis component antigens (PT, FHA e PRN), according to the blood sampling time point.

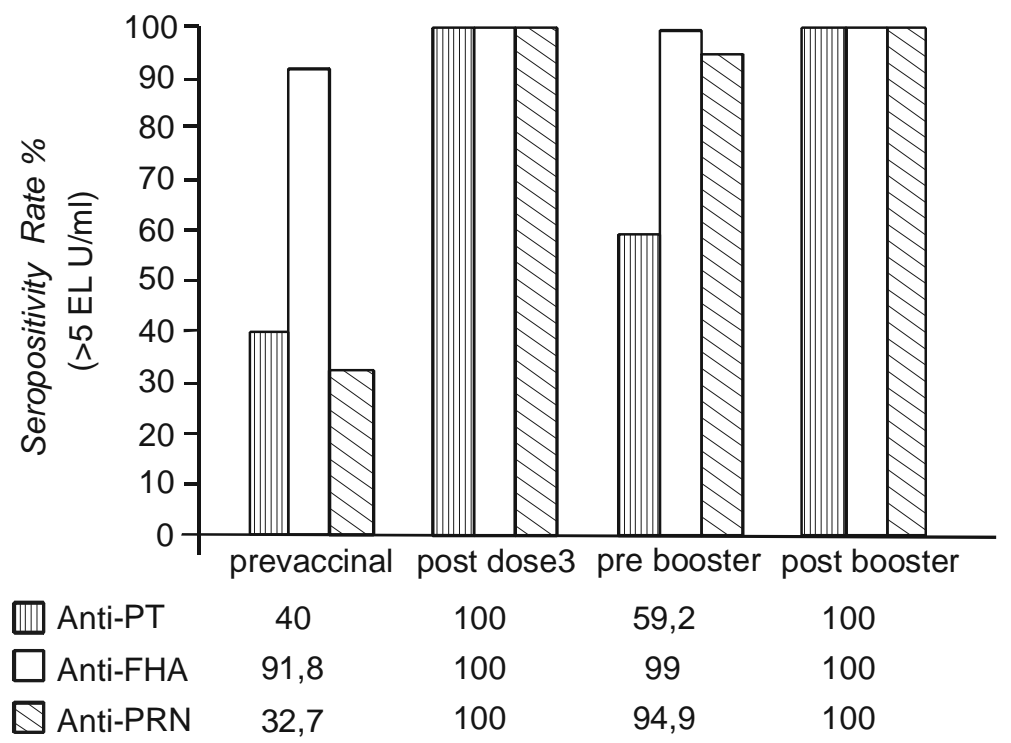

Figure 4. Seropositivity rate for Hepatitis B surface antigen (HbsAg), according to the blood sampling time point.

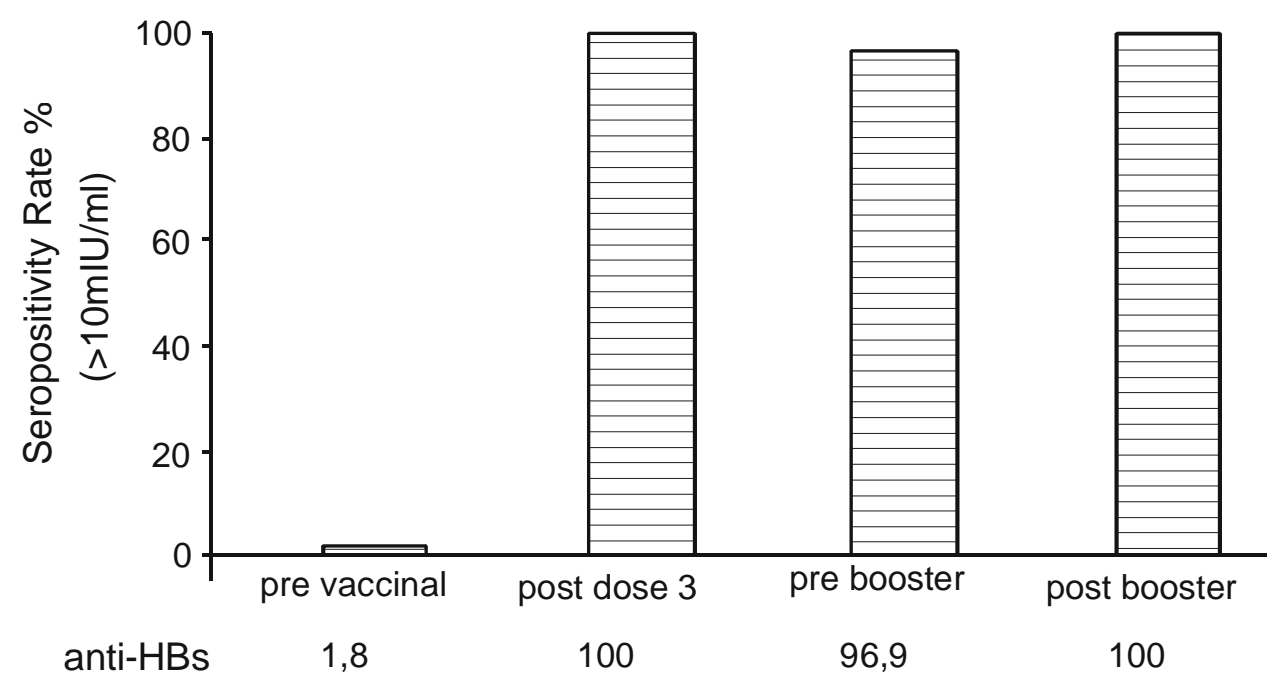


Figure 5. Proportion of subjects with titers greater than $0.15 \mu \mathrm{g} / \mathrm{mL}$ and $1 \mu \mathrm{g} / \mathrm{mL}$ for anti-PRP, according to the blood sampling time point.

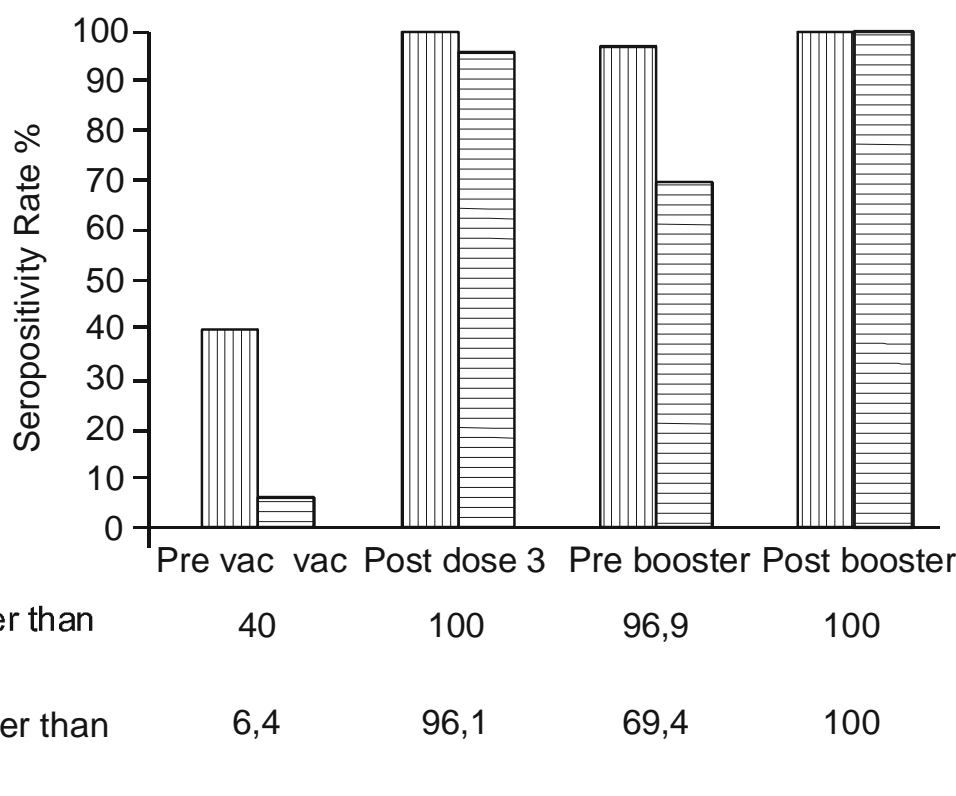

Table 1. Geometric mean titer for antibodies against the enrolled vaccine antigens, according to the blood sampling time point

\begin{tabular}{lcccc}
\hline & \multicolumn{4}{c}{ Blood Sampling Time point } \\
\cline { 2 - 5 } Antigen & Pre vaccine & Post dose 3 & Pre booster & Post booster \\
& GMT & GMT & GMT & 5.01 \\
\hline Diphtheria(IU/mL) & 0.3 & 1.3 & 0.08 & $(4.07-6.17)$ \\
CI (95\%) & $(0.22-0.40)$ & $(1.20-1.54)$ & $(0.07-0.08)$ & 14.18 \\
Tetanus (IU/mL) & 0.25 & 3.62 & 0.48 & $(11.85-16.98)$ \\
CI (95\%) & $(0.18-0.34)$ & $(3.09-4.24)$ & $(0.39-0.58)$ & 116.3 \\
PT (EL U/mL) & 4.4 & 55.1 & 5.1 & $(98.1-138.0)$ \\
CI (95\%) & $(3.8-5.2)$ & $(49.0-62.0)$ & $(4.4-5.8)$ & 851.5 \\
FHA (EL U/mL) & 18.5 & 290.8 & 38.3 & $(753.9-961.7)$ \\
CI (95\%) & $(15.6-21.9)$ & $(254-333.0)$ & $(32.4-45.3)$ & 595.7 \\
PRN (EL U/mL) & 4.1 & 183.1 & 16.8 & $(496.5-714.8)$ \\
CI (95\%) & $(3.5-4.8)$ & $(158.2-211.9)$ & $(14.3-19.9)$ & 5093.17 \\
HBs (mIU/mL) & 5.24 & 1328.52 & 107.69 & $(3,725.52-6,962.90)$ \\
CI (95\%) & $(4.84-5.67)$ & $(1,037.57-1,701.04)$ & $(79.98-145.01)$ & 129.1 \\
PRP (mg/mL) & 0.15 & 18.59 & 2.15 & $(96.84-172.11)$ \\
CI (95\%) & $(0.12-0.19)$ & $(13.71-25.22)$ & $(1.60-2.88)$ & \\
\hline
\end{tabular}

$\mathrm{CI}=$ Confidence intervals. GMT = Geometric mean antibody titer. IU = International units. EL U/mL = ELISA units per milliliter. 
Table 2. Relation between the post booster geometric mean antibody titer (GMT) and the post basic vaccination GMT and between the post booster GMT and the pre booster GMT according to the antibody

\begin{tabular}{lcc}
\hline Antibody & $\begin{array}{c}\text { GMT Post booster / } \\
\text { GMT Post basic vaccination }\end{array}$ & $\begin{array}{c}\text { GMT Post booster / } \\
\text { GMT Pre booster }\end{array}$ \\
\hline Diphtheria antitoxin & 3.9 & 62.6 \\
Tetanus antitoxin & 3.9 & 30.2 \\
Anti-PT & 2.1 & 22.8 \\
Anti-FHA & 2.9 & 22.2 \\
Anti-PRN & 3.3 & 35.5 \\
Anti-HBs & 3.8 & 47.3 \\
Anti-PRP & 6.9 & 60.2 \\
\hline
\end{tabular}

Table 3. Geometric mean antibody titers (in EL U/mL) to the three Pertussis antigens after basic vaccination, compared with other studies

\begin{tabular}{lccc}
\hline Studies & \multicolumn{3}{c}{ GMT(EL U/mL) } \\
& anti-PT & anti-FHA & anti-PRN \\
\hline This study & 55.1 & 290.8 & 183.1 \\
Greenberg et al., 2002 [4] & 68.8 & 501 & 205 \\
Yeh et al., 2001 [16] & 72.8 & 234.3 & 154.9 \\
Giammanco et al., (1998) [21] & 56.1 & 153 & 240 \\
\hline GMT = Geometric mean antibody titer. EL U/mL = ELISA units per milliliter.
\end{tabular}

The seropositivity rate for the Polyribosyl-RibitolPhosphate (PRP) antigen was expressed using two different values (Figure 5). In addition to the cut-off value of $0.15 \mu \mathrm{g} / \mathrm{mL}$, which gives an estimation of the minimum level for protection, the seropositivity rate with a level of anti-PRP antibodies greater than or equal to $1 \mathrm{mg} / \mathrm{mL}$ was also calculated. This latter gives an estimate for long-term protection.

When the GMTs for the diphtheria, tetanus, PT, FHA, PRN, HBs and PRP antigens (Table 1 ) were compared, all the vaccine components were found to present a similar evolution, with a marked rise in antibody titers after the basic scheme, followed by a decline over the period immediately preceding the booster dose. After this, there was a new increase in antibodies, which exceeded the concentrations reached after the basic scheme by a factor of two to seven (Table 2).

\section{Discussion}

Comparison of the results from this study with those from other studies that utilized the same vaccines or their components helps comprehend the immunogenicity of the vaccines. Before the first immunization, the anti-diphtheria and anti-tetanus antibodies presented high levels (seropositivity rate of around $60 \%$ for both antibodies). This was probably the result of passage through the placenta and thus reflects the mother's state of vaccination. After the basic scheme, there was a rise in the antibody titers. The GMT for the anti-diphtheria and anti-tetanus antibodies (respectively, 1.3 and $3.6 \mathrm{IU} / \mathrm{mL}$ ) were similar to those found in an American study for the same vaccine (respectively, 1.81 and $3.44 \mathrm{IU} / \mathrm{mL}$ ) [4]. The study by Yeh et al. (2001), for the same vaccine combination 
and simultaneous application of inactivated polio vaccine (IPV) and PRP-T vaccines, also reported similar results, with GMT of $1.2 \mathrm{IU} / \mathrm{mL}$ for anti-diphtheria antibodies and $2.3 \mathrm{IU} / \mathrm{mL}$ for anti-tetanus antibodies, and all of the vaccinated patients attained a protective seropositivity rate after completing the basic immunization scheme, as we also found in our study [16]. There was a decline in anti-diphtheria and antitetanus antibody titers before the booster dose. The values obtained(GMT of $0.08 \mathrm{IU} / \mathrm{mL}$ and seropositivity rate of $33.7 \%$ for diphtheria, and GMT of $0.47 \mathrm{IU} / \mathrm{mL}$ and seropositivity rate of $93.9 \%$ for tetanus) were similar to those obtained in another study [16], which found a GMT of $0.1 \mathrm{IU} / \mathrm{mL}$ and a seropositivity rate of $64.4 \%$ for diphtheria, and a GMT of $0.5 \mathrm{IU} / \mathrm{mL}$ and a seropositivity rate of $100 \%$ for tetanus; these authors utilized the same serological test (ELISA). To determine the negative anti-diphtheria antibody titers, by ELISA, the authors [16] utilized the Vero cell neutralization test; when these two tests (ELISA and Vero cell neutralization) were used, the seropositivity rate rose from $64.4 \%$ to $90.3 \%$. This suggests that the large fall in anti-diphtheria antibodies observed immediately prior to the booster dose was mainly due to the serological method utilized, since the neutralization test has greater sensitivity for detecting the immunological response for diphtheria when the antibody titer is less than $0.1 \mathrm{IU} /$ $\mathrm{mL}$ and therefore ELISA underestimates the seroprotection rate for this group [17]. The large increase in concentrations of these two antibodies that occurred after the booster dose, in relation to the concentrations found immediately before this dose, was also found in an American study [16]. However, in that study, the GMT findings for both the anti-diphtheria antibodies (5.01 IU/mL vs. $2.0 \mathrm{IU} / \mathrm{mL}$ ) and the antitetanus antibodies $(14.18 \mathrm{IU} / \mathrm{mL}$ vs. $6.1 \mathrm{IU} / \mathrm{mL})$ were about twice the level that we found. However, in both studies the seropositivity rates became $100 \%$ for both antibodies, and the GMT values after the booster dose exceeded the pre-booster values by a factor of at least 12.

We examined the GMTs of the three antigens that made up the pertussis component (PT, FHA, PRN) after the basic vaccination schedule scheme their and compared them with the values found in other studies (Table 3). The differences found in comparison with these other studies, which utilized the same vaccine and the same serological tests, may be due to variation in the antibody response to the antigens in different populations. The relevance of the differences found diminishes when it is considered that the seropositivity rate was $100 \%$ for the three vaccine antigens, both in our study and in the three other studies with which it was compared.

Differing from the other antigens for which minimum levels of protection have been established, there is currently no laboratory measurement for immunogenicity that can be correlated with protective efficacy for the antigens of the pertussis component [5]. Consequently, the immunogenicity of the combined vaccine after the basic scheme in our study was compared with the immunogenicity found in a multicenter study of acellular pertussis vaccines (MAPT) promoted by the American National Institute of Allergy and Infectious Diseases. The latter was developed using the same diphtheria-tetanus-acellular pertussis vaccine that serves as basis for our combination vaccine. A field study in Italy demonstrated 84\% efficacy for this DTPa vaccine [18]. Since the GMT were similar to (55.1 U EL/mL vs. $54 \mathrm{U} \mathrm{EL/mL}$ for the anti-PT antibody and $183.1 \mathrm{U}$ EL/mL vs. 185 $\mathrm{U} E \mathrm{E} / \mathrm{mL}$ for the anti-PRN antibody) or greater than (290.8 U EL/mL vs. $103 \mathrm{U}$ EL/mL for the anti-FHA antibody) those obtained in the multicenter study [19], the comparison of the results demonstrates that the combination of the DTPa (diphtheria-tetanus-acellular pertussis) vaccine with the vaccine against hepatitis $B$ did not result in decreased immunogenicity of the pertussis component of this combination, when compared to the uncombined DTPa vaccine. Consequently, this combination did not interfere in vaccine efficacy.

At the time of the booster dose, the titers of the three antibodies of the pertussis component presented a large fall in relation to the titers found right after the basic scheme. When compared with another study, for the same vaccine, it was found that the titers were lower for the three antibodies (anti-PT 5.1 vs. 8.4 U EL/mL; 
anti-FHA 38.3 vs. 49 U EL/mL; and anti-PRN 16.8 vs. $25 \mathrm{UEL} / \mathrm{mL}$ ) [16]. However, when the results from the post-booster dose were compared with those from the other study [16], the titers were higher for the three antibodies (anti-PT 116.3 vs. $100 \mathrm{U}$ EL/mL; anti-FHA 851.5 vs. $280 \mathrm{U}$ EL/mL; and anti-PRN 595.7 vs. 303 $\mathrm{U}$ EL/mL) [16]. The children who did not present any seropositivity for one of the pertussis antigens before the booster dose responded to the booster dose. The large rise in GMTs for the three pertussis antigens in relation to the levels prior to this dose, indicates effective previous sensitization and immunological memory.

When hepatitis B applied as avaccine component was compared with vaccine against hepatitis B applied separately in the same vaccination scheme, the GMT obtained after the basic scheme $(1,328.52 \mathrm{mIU} / \mathrm{mL})$ was less than was obtained by Greenberg et al., in 1996 $(1,812 \mathrm{mIU} / \mathrm{mL})$, and greater than was obtained by Yeh et al., in 2001 (805 mIU/mL), with a seropositivity rate similar to what was found in the two studies (99\% and $100 \%)$ [16,20]. In comparison with other studies that involved the same combined vaccine after the basic scheme, the anti-HBs GMT was greater than what was obtained by Giammanco et al. ( $949 \mathrm{mIU} / \mathrm{mL})$, Yeh et al. (919 mIU/mL) and Greenberg, in 2002 (1052 mIU/ $\mathrm{mL})[4,16,21]$. Although the need for a booster dose is a subject for debate [22], it is certain that no booster dose would be needed during a period of 9-12 months after the third vaccine dose. The application of such a dose would have the objective of assessing the immunological memory triggered by the antigen stimulus. The rise in the GMT for anti-HBs to more than 5000 $\mathrm{mIU} / \mathrm{mL}$ and the approximately 50 -fold increase in the GMT for anti-HBs after the booster dose, in comparison with the pre-booster period, demonstrates that there is a memory response.

One difficulty in including the vaccine against hepatitis $\mathrm{B}$ as a component of a combined vaccine is in the adaptation to the vaccination scheme. This may be adapted provided that it does not alter the immunological response [3,4]. When the vaccine against hepatitis $B$ is applied in the most traditional scheme (at birth and at the ages of one and six months), the GMT is greater than in the scheme of applying at the ages of two, four and six months, although the antibody titers are also high and all the children are protected against clinical disease and chronic infection. The difference in vaccine response found between the two schemes can be explained by the well-known phenomenon in which when the interval between the second and third vaccine doses is increased, higher levels of anti-HBs are attained [23].

Another question that must be addressed regarding the vaccine against hepatitis $B$ is the age at which the vaccination should start. The World Health Organization (WHO) recommends that, in countries with high levels of perinatal transmission, a first dose of the vaccine against hepatitis $B$ should be given within the first 12 hours of life [24]. Studies have demonstrated that a vaccine combination that includes the vaccine against hepatitis B can be administered after one dose of the vaccine against hepatitis $B$ given at birth, without increasing reactogenicity and with increased anti-HBs antibodies [25,26]. On the basis of these data, a vaccination scheme can be developed, consisting of one dose of vaccine against hepatitis $\mathrm{B}$, applied at birth, followed by the combined doses at two, four and six months, or alternatively without the need for the component of the vaccine against hepatitis $B$ at the age of four months.

When the response to the vaccine against Haemophilus influenzae type b (Hib) was evaluated, it was found that after the basic scheme, the GMT for antiPRP antibodies $(18.59 \mathrm{mcg} / \mathrm{mL}$ ) was around two to three times greater than the means observed in other studies that also utilized anti-PRP vaccine conjugated with tetanus toxoid that was applied concomitantly with DTPa-HB vaccine, albeit manufactured by another laboratory (Aventis-Pasteur): $6.26 \mathrm{mcg} / \mathrm{mL}$ [27], $7.1 \mathrm{mcg} / \mathrm{mL}$ [16] and $9.8 \mathrm{mcg} / \mathrm{mL}$ [4]. Between nine and twelve months after the conclusion of the basic scheme (pre-booster period), the GMT was greater than was observed in another study ( $2.15 \mathrm{mcg} / \mathrm{mL} v \mathrm{~s} .1 .2 \mathrm{mcg} / \mathrm{mL})$ as was the seropositivity rate for anti-PRP concentrations greater than $1 \mathrm{mcg} / \mathrm{mL}$ (69.4\% vs. 60\%) [16]. In both studies, after the booster dose, the seropositivity rate for anti-PRP concentrations greater than $1 \mathrm{mcg} / \mathrm{mL}$ was $100 \%$, although the GMT was around five times greater in our study (129.1 mcg/mL vs. $27 \mathrm{mcg} / \mathrm{mL})$ [16]. 
In a comparison of the concentrations of anti-PRP antibodies found after applying the basic vaccination scheme concomitantly, with another study in which the vaccine against Hib was administered in combination with the DTPa vaccine and the vaccine against hepatitis B (DTPa-HB/Hib), a large difference in anti-PRP concentration was found $(18.59 \mathrm{mcg} / \mathrm{mL} v \mathrm{ss} .4 .05 \mathrm{mcg} /$ $\mathrm{mL})$ [28]. This difference was already expected, since large numbers of reports have noted a marked reduction in the response to PRP following primary immunization. This has been observed with most of the vaccine combinations in the acellular pertussis vaccine and the vaccine against Hib (DTPa-Hib). The mechanisms involved in this interaction, and also the clinical repercussions coming from this reduction, are still polemical points and motives for discussion within the scientific community [5,29,30].

It is therefore concluded that the concomitant administration of the combined DTPa-HB vaccine with the anti-Haemophilus influenzae type b vaccine induces a powerful antibody response to all the antigen components. The antibody titers for all the vaccine components presented a large rise after the basic scheme, followed by a decline within the period immediately prior to the booster dose. After this, a new increase in antibodies was found, and this exceeded the titers attained after the basic scheme by a factor of two to seven times, thereby showing immunological memory. The seropositivity rate after the basic scheme was $100 \%$ for all the antigens (diphtheria, tetanus, PT, FHA, PRN, HBs and PRP). Before the booster dose, all the antigens had seropositivity rates of more than 94\%, except for diphtheria, which underwent a large fall and reached $33.7 \%$, and pertussis toxin with a rate of $59 \%$. After the booster dose, the seropositivity rate once again became $100 \%$ for all antigens. These vaccines were immunogenic both after the primary immunization and after the booster dose.

\section{Acknowledgments}

We thank all of the patients who enrolled in this study. Financial support was provided by SmithKline Beecham Biologicals.

\section{References}

1. Plotkin S.L., Plotkin S.A. A short history of vaccination. In: Plotkin S.A., Orenstein W.A. (eds.). Vaccines. $3^{\text {rd }}$ ed. Philadelphia: Saunders; 1999. p.1-8.

2. Decker M.D., Edwards K.M., Steinhoff M.C., et al. Comparison of 13 acellular pertussis vaccines: adverse reactions. Pediatrics 1995;96:557-66.

3. Pines E., Barrand M., Fabre P., Et al. New acellular pertussis-containing paediatric combined vaccines. Vaccine 1999;17:1650-6.

4. Greenberg D.P., Wong V.K., Partridge S., et al. Safety and immunogenicity of a combination diphtheria-tetanus toxoids-acellular pertussis-hepatitis B vaccine administered at two, four and six months of age compared with monovalent hepatitis B vaccine administered at birth, one month and six months of age. Pediatr Infect Dis J 2002;21:769-76.

5. Edwards K.M., Decker M.D., Mortimer E. A. JR. Pertussis Vaccine. In: Plotkin S.A., Orenstein W.A., Vaccines. $3^{\text {rd }}$ ed. Philadelphia: Saunders; 1999. p. 293-343.

6. Hargreaves R.M., Slack M.P.E., Howard A.J., et al. Changing patterns of invasive Haemophilus influenzae disease in England and Wales after introduction of the Hib vaccination programme Br Med J 1996;312:160-1.

7. Peltola H., Aavitsland P., Hansen K.G., et al. Perspective: a five-country analysis of the impact of four different Haemophilus influenzae type b conjugates and vaccination strategies in Scandinavia. J Infect Dis 1999;179(1):223-9.

8. Decker M.D., Edwards K.M. Combination Vaccines. In: Plotkin S.A., Orenstein W.A.(eds.). Vaccines. $3^{\text {rd }}$ ed. Philadelphia: Saunders; 1999. p. 508-25.

9. Christy C., Pichichero M.E., Reed G.F., et al. Effect of gender, race, and parenteral education on immunogenicity and reported reactogenicity of acellular and whole-cell pertussis vaccines. Pediatrics 1995;96:584-7.

10. Martins R.M. Infecções por Haemophilus influenzae. In: Farhat C.K., Carvalho E.S., Carvalho L.H.F.R., Succi R.C.M. Infectologia Pediátrica. São Paulo: Ateneu; 1998. p.268-80.

11. Kanra G., Ceyhan M., Ecevit Z., et al Primary vaccination of infants with a combined diphtheria-tetanus-acellular pertussis-hepatitis B vaccine. Pediatr Infect D J 1995;14(11):998-1000.

12. Melville-Smith M.E., Seagroatt V.A., Watkins J.R. A comparation of enzyme linked immunosorbent assay (ELISA) with the toxin neutralization test in mice as a method for the estimation of tetanus antitoxin in human sera. J Biol Stand 1983;11:137-44.

13. Ruuskanen O., Noel A., Putto-Laurila A., et al. Development of an acellular pertussis vaccine and its administration as a booster in healthy adults. Vaccine 1991;9:117-21. 
14. Plotkin S.A. Immunologic correlates of protection induced by vaccination. Pediatr Infect Dis J 2001;20:63-75.

15. Colucci A. Reatogenicidade da vacina combinada DTPaHBV (tríplice acelular-hepatite B) e da vacina contra Haemophilus influenzae tipo b quando aplicadas simultaneamente. [M.Sc. thesis]. São Paulo: Universidade Federal de São Paulo; 2001.

16. Yeh S.H., Ward J.I., Partridge S., et al. Safety and immunogenicity of a pentavalent diphtheria, tetanus, pertussis, hepatitis $B$ and polio combination vaccines in infants. Pediatr Infect Dis J 2001;20:973-80.

17. Gupta R.K., Griffin P., Xu J., et al. Diphtheric anti-toxin levels in U.S. blood and plasma donors. J. Infect Dis 1996;173(6):1493-7.

18. Greco D., Salmaso S., Mastrantonio P., et al. A controlled trial of two acellular vaccines and one whole-cell vaccine against pertussis. N Engl J Med 1996;334:341-8.

19. Edwards K.M., Decker M.D., Steinhoff M.C., et al. Comparison of 13 acellular pertussis vaccines: Overview and serologic response. Pediatrics 1995;96:548-57.

20. Greenberg D.P., Vadheim C.M., Marcy S.M., et al. Safety and immunogenicity of a recombinant hepatitis $\mathrm{B}$ vaccine administered to infants at 2,4 and 6 months of age. Vaccine 1996;14:811-6.

21. Giammanco G., Moiraghi A., Zotti C., et al. Safety and immunogenicity of a combined diphtheria-tetanusacellular pertussis-hepatitis B vaccine administered according to two different primary vaccination schedules. Vaccine 1998;16:722-6.

22. Mahoney, F.J. Kane M. Hepatitis B Vaccine. In: Plotkin S.A., Orenstein W.A. (eds.). Vaccines. $3^{\text {rd }}$ ed. Philadelphia: Saunders; 1999. p.158-82.

23. Greenberg D.P. Considerations for hepatitis B as part of a combination vaccine. Pediatr Infect Dis J 2001;20:34-9.

24. World Health Organization. The Children's Vaccine Initiative and the Global Programme for Vaccine and Immunization recommendations from the Special Advisory Group of Experts. Wkly Epidemiol Rec 1996;71:261-8.

25. Gylca R., Gylca V., Benes O., et al. A new DTPa-HBV-IPV vaccine co-administered with Hib, compared to a commercially available DTPw-IPV/Hib vaccine coadministered with HBV, given at 6, 10 and 14 weeks following $\mathrm{HBV}$ at birth. Vaccine 2001;19:825-33.

26. Pichichero M.E., Blatter M.M., Reisinger K.S., et al. Impact of a birth dose of hepatitis B vaccine on the reactogenicity and immunogenicity of diphtheria-tetanus-acellular pertussis-hepatitis B-inactivated poliovirusHaemophilus influenzae type b combination vaccination. Pediatr Infect Dis J 2002;21:854-9.
27. Greenberg D.P., Wong V.K., Partridge S., et al. Immunogenicity of Haemophilus influenzae type btetanus toxoid conjugate vaccine when mixed with a diphtheria-tetanus-acellular pertussis-hepatitis B combination vaccine. Pediatr Infect Dis J 2000;19:1135-40.

28. Arístegui J., Dal-Ré R., Garrote E., et al. Assessment of the immunogenicity and reactogenicity of a quadrivalent diphtheria, tetanus, acellular pertussis and hepatitis B (DTPa-HBV) vaccine administered in a single injection with Haemophilus influenzae type b conjugate vaccine, to infants at 2, 4 and 6 months of age. Vaccine 1998; 16:1976-81.

29. Granoff D.M. Challengers for licensure of new diphtheria, tetanus, acellular pertussis (DtaP) combination vaccines: point. Pediatr Infect Dis J 1996;15:1069-70.

30. Edwards K.M., Decker M.D. Challengers for licensure of new diphtheria, tetanus toxoid, acellular pertussis (DtaP) combination vaccines: counterpoint. Pediatr Infect Dis J 1996;15:1070-3. 